\title{
ON TENSOR INDUCTION OF GROUP REPRESENTATIONS
}

\author{
L. G. KOVÁCS \\ Communicated by $\mathbf{H}$. Lausch \\ Dedicated to G. E. (Tim) Wall, in recognition of his distinguished contribution to \\ mathematics in Australia, on the occasion of his retirement
}

\begin{abstract}
Let $G$ be a (not necessarily finite) group and $\rho$ a finite dimensional faithful irreducible representation of $G$ over an arbitrary field; write $\bar{\rho}$ for $\rho$ viewed as a projective representation. Suppose that $\rho$ is not induced (from any proper subgroup) and that $\bar{\rho}$ is not a tensor product (of projective representations of dimension greater than 1 ). Let $K$ be a noncentral subgroup which centralizes all its conjugates in $G$ except perhaps itself, write $H$ for the normalizer of $K$ in $G$, and suppose that some irreducible constituent, $\sigma$ say, of the restriction $\rho \downarrow_{K}$ is absolutely irreducible. It is proved that then ( $\rho$ is absolutely irreducible and) $\bar{\rho}$ is tensor induced from a projective representation of $H$, namely from a tensor factor $\pi$ of $\bar{\rho} \downarrow_{H}$ such that $\pi \downarrow_{K}=\bar{\sigma}$ and $\operatorname{ker} \pi$ is the centralizer of $K$ in $G$.
\end{abstract}

1980 Mathematics subject classification (Amer. Math. Soc.) (1985 Revision): Primary 20 C 15 , $20 \mathrm{C} 20$.

\section{Introduction}

All representations considered in this paper will be finite dimensional representations over fields. For each representation $\rho: G \rightarrow G L(d, \mathbb{F})$ of a group $G$ (over an arbitrary field $\mathbb{F}$ ), let $\bar{\rho}: G \rightarrow P G L(d, \mathbb{F}$ ) denote the 'projective representation' obtained by composing $\rho$ with the natural map of $G L(d, \mathbb{F})$ onto $\operatorname{PGL}(d, \mathbb{F})$.

Let $\rho$ be a faithful irreducible representation of a (not necessarily finite) group $G$ over an algebraically closed field (of arbitrary characteristic), and $M$ a noncentral normal subgroup. As is well known, Clifford's Theorem [4, $11.1]$ yields that if $\rho$ is not induced (from any proper subgroup) then the restriction $\rho \downarrow_{M}$ is a direct sum of pairwise equivalent irreducible represen-

(C) 1990 Australian Mathematical Society $0263-6115 / 90 \$ A 2.00+0.00$ 
tations. If moreover $\bar{\rho}$ is not a tensor product (of projective representations of dimension greater than 1), then another theorem of Clifford $[4,11.20]$ gives that $\rho \downarrow_{M}$ itself is irreducible. The aim of this paper is to take one more step by showing that if in this setting $\bar{\rho}$ is not tensor induced (from any projective representation of any proper subgroup), then $M$ cannot be written (nontrivially) as a central product of a conjugacy class of subgroups of $G$. For example, $M$ cannot be a finite nonabelian nonsimple minimal normal subgroup.

For the motivating application, let $G$ be a finite nonabelian group with centre $Z$ and generalized Fitting subgroup $F$ (see [7, X.13]), and suppose that $G$ has a faithful primitive representation $\rho$ (over an algebraically closed field) such that $\bar{\rho}$ is neither tensor induced nor a tensor product (in any nontrivial way). Then either $F / Z$ is a simple group or it is a faithful irreducible $G / F$-module, a module which carries a nondegenerate symplectic form respected by the action of $G / F$ and which is not 'form-induced' from any proper subgroup of $G / F$. By a result of Berger [1, Theorem 7.8], in the latter case $F / Z$ is a 'minimal module': that is, viewed as a module for any normal subgroup of $G / F$, it is either homogeneous or a direct sum of two homogeneous components each of which is totally isotropic. Thus after induction, tensor factorization, and tensor induction have been exploited, what is left is either a 'nearly' simple group or a situation very similar to one which has been extensively studied in the context of primitive linear groups with an extraspecial normal subgroup.

The principal result is both more general and more positive than the concise version stated above; it is given (in two versions) in Section 6. A number of examples in Section 8 mark some of the boundaries of this approach.

REMARK (added 24 May 1990). I have learned that the main result of this paper has also been obtained by Professor Peter Schmid in his paper "Cohomology of tensor induction' (to appear in J. Algebra). The two treatments are complementary, in that each explores several aspects of the problem which are not dealt with by the other.

\section{Projective representations}

The reader will have noted that projective representations are here viewed simply as homomorphisms into some $\operatorname{PGL}(d, \mathbb{F})$ : in the present context, there seems to be no advantage in the tradition of working with the not necessarily homomorphic maps into $G L(d, \mathbb{F})$ which yield these homomorphisms upon composition with the natural map of $G L(d, \mathbb{F})$ onto $P G L(d, \mathbb{F})$. The word 'representation' without the adjective 'projective' will always mean a 
homomorphism to a $G L(d, \mathbb{F})$; for the sake of emphasis, these homomorphisms will at times be referred to as genuine representations.

It may be as well to review some general facts in terms of these conventions. The only projective representation of dimension 1 is now the trivial one. A projective representation $\pi: G \rightarrow P G L(d, \mathbb{F})$ is said to be (absolutely) irreducible if the complete inverse image of $G \pi$ in $G L(d, \mathbb{F})$ is an (absolutely) irreducible linear group. Two projective representations are called equivalent if their dimensions are equal and one is the composite of the other with an inner automorphism of the relevant $P G L(d, \mathbb{F})$. Given two genuine representations $\rho$ and $\sigma$, it is easy to see that the corresponding projective representations $\bar{\rho}$ and $\bar{\sigma}$ are equivalent if and only if $\rho$ is equivalent to the tensor product of $\sigma$ with a 1-dimensional representation. Thus while the equivalence type of the direct sum $\rho \oplus \sigma$ depends only on the equivalence types of $\rho$ and $\sigma$, in general the equivalence type of $\overline{\rho \oplus \sigma}$ is not determined by the equivalence types of $\bar{\rho}$ and $\bar{\sigma}$. This is just a small part of the reason why there is no sensible way to define direct sums of projective representations; the real reason is, of course, that the 'block diagonal' inclusion of the (abstract, external) direct product $G L(c, \mathbb{F}) \times G L(d, \mathbb{F})$ in $G L(c+d, \mathbb{F})$ yields no homomorphism from $P G L(c, \mathbb{F}) \times P G L(d, \mathbb{F})$ into $P G L(c+d, \mathbb{F})$. Accordingly, for projective representations one cannot define induction either.

There is no such difficulty with tensor products: the Kronecker product homomorphism of $G L(c, \mathbb{F}) \times G L(d, \mathbb{F})$ into $G L(c d, \mathbb{F})$ does yield a homomorphism, indeed an embedding, of $P G L(c, \mathbb{F}) \times P G L(d, \mathbb{F})$ into $P G L(c d, \mathbb{F})$. One can therefore define the tensor product of two projective representations; the equivalence type of the tensor product depends only on the equivalence types of the tensor factors, and the formation of tensor products commutes with the map $\rho \mapsto \bar{\rho}$ from genuine representations to projective representations.

Similarly, the $n$-fold Kronecker product yields a homomorphism, $\kappa$ say, of the $n$-fold direct power of $G L(d, \mathbb{F})$ into $G L\left(d^{n}, \mathbb{F}\right)$. It is not hard to see that there is a copy of the symmetric group $S_{n}$ in the group of all permutation matrices in $G L\left(d^{n}, \mathbb{F}\right)$ whose conjugation action on the image of this embedding corresponds to permuting the Kronecker factors. Thus if the $n$-fold direct power of $G L(d, \mathbb{F})$ is regarded as the base group of the (permutational) wreath product $G L(d, \mathbb{F}) \mathrm{Wr} S_{n}$, this $\kappa$ extends to a homomorphism $\kappa^{+}: G L(d, \mathbb{F})$ Wr $S_{n} \rightarrow G L\left(d^{n}, \mathbb{F}\right)$, mapping the top group of the wreath product to the copy of $S_{n}$ mentioned above. Let $H$ be a subgroup of index $n$ in a group $G$, and $\rho: H \rightarrow G L(d, \mathbb{F})$ a representation of $H$. One may paraphrase [4, Definition 13.5] as follows: the rep- 
resentation $\rho \uparrow^{\otimes G}$ tensor induced from $\rho$ is the composite of a certain embedding $\varphi: G \rightarrow H$ Wr $S_{n}$ (defined in [4, 13.3]), the homomorphism $H$ Wr $S_{n} \rightarrow G L(d, \mathbb{F})$ Wr $S_{n}$ obtained from $\rho$ in the obvious manner, and $\kappa^{+}$. As in Berger [1], this $\varphi$ will be called a Frobenius embedding, the relevant homomorphism $H$ Wr $S_{n} \rightarrow G L(d, \mathbb{F})$ Wr $S_{n}$ will be written as $\rho \mathrm{Wr} S_{n}$. Recall that $\varphi$ depends on the choice of representatives for the cosets of $G$ modulo $H$, but only up to composition with inner automorphisms of its codomain, so the equivalence type of $\rho \uparrow^{\otimes G}$ depends only on that of $\rho$.

There is a corresponding embedding $\tilde{\kappa}: P G L(d, \mathbb{F})$ Wr $S_{n} \rightarrow P G L\left(d^{n}, \mathbb{F}\right)$. Given a projective representation $\pi: H \rightarrow P G L(d, \mathbb{F})$, one calls the composite $\varphi\left(\pi \mathrm{Wr} S_{n}\right) \widetilde{\kappa}$ the projective representation $\pi \uparrow^{\otimes G}$ of $G$ tensor induced from $\pi$. This is consistent with the definition of tensor induction of projective representations given by Berger in [1,2B] (but beware of confusion with Definition 5.8 of that paper: the explanation for the connection with that definition is in the second paragraph on page 25 of [1]). It is clear that the equivalence type of $\pi \uparrow^{\otimes G}$ depends only on that of $\pi$, and also that $\overline{\rho \uparrow^{\otimes G}}$ is equivalent to $\bar{\rho} \uparrow^{\otimes G}$.

It will pay to pursue the analogy one step further: the (genuine) representation $\rho \uparrow^{G}$ of $G$ induced from $\rho$ in the usual (additive) sense is easily seen to be the composite homomorphism obtained as $\varphi$ followed by $\rho \mathrm{Wr} S_{n}$ followed by the usual 'block monomial' embedding of $G L(d, \mathbb{F})$ Wr $S_{n}$ in $G L(d n, \mathbb{F})$.

REMARKS. It is customary but not entirely justified to talk about 'the' representation of a group induced or tensor induced from a given representation of a subgroup, a custom implicit in any notation such as $\rho \uparrow^{G}$ or $\pi \uparrow^{\otimes G}$. As has been seen, the equivalence type of the induced or tensor induced representation is indeed well defined; however, in general there is no canonical choice of a representative of that equivalence type. Such a choice would depend not only on coset representatives but also on a linear order for the set of cosets and on appropriate further conventions. Some of these difficulties have been masked in the above discussion by speaking of 'the' embedding $\widetilde{\kappa}$, of 'the' block monomial embedding, and so on, as if these were unique or at least canonically chosen. (For induction or co-induction of genuine representations, module language and tensoring with the group algebra or taking a Hom set provide natural, choice-free, functorial alternatives, but so far I have not seen a choice-free definition for a tensor induced module.)

A related abuse of language enshrined in tradition is the failure to acknowledge that while the various representations of a group induced from a given representation of a subgroup are all equivalent, they need not form a 
complete equivalence class. It is very convenient here, too, to say simply that a representation is induced or tensor induced when one means only that it is equivalent to at least one induced or tensor induced representation.

\section{Wreath products}

Whether we are dealing with a tensor induced projective representation or with an (additively) induced genuine representation, the image lies in a wreath product with top group $S_{n}$. By construction, each wreath product is the semidirect product of its base group and top group, and so comes with a 'top projection': an idempotent endomorphism with image the top group and kernel the base group. Since $G$ acts transitively on its set of cosets modulo $H$, its image in the relevant wreath product is mapped by this top projection onto a transitive subgroup of $S_{n}$. This observation has a useful converse, which is an easier variant of the 'if' part of $\left[9\right.$, Theorem $\left.1^{\prime}\right]$.

LeMMA. Given any homomorphism $\gamma: G \rightarrow A$ Wr $S_{n}$ such that the top projection maps $G \gamma$ onto a transitive subgroup of $S_{n}$, there is a subgroup $H$ (of index $n$ in $G$ ) and a homomorphism $\alpha: H \rightarrow A$ such that $\gamma$ is a Frobenius embedding $\varphi: G \rightarrow H \mathrm{Wr} S_{n}$ followed by $\alpha \mathrm{Wr} S_{n}$ followed by an inner automorphism of $A \mathrm{Wr} S_{n}$.

Proof. Call that top projection $\tau$, let $S_{n-1}$ be a point stabilizer in $S_{n}$, and $H$ the complete inverse image $S_{n-1}(\gamma \tau)^{-1}$. Since $G \gamma \tau$ is transitive, this choice of $H$ already ensures that $\varphi\left(\alpha \mathrm{Wr} S_{n}\right) \tau$ and $\gamma \tau$ can differ only by an inner automorphism of $S_{n}$, regardless of how $\varphi$ and $\alpha$ may be chosen. Differently put, to each choice of $\varphi$ and $\alpha$, conjugation by a suitable element of the top group defines an inner automorphism of the wreath product, $\sigma$ say, such that $\varphi\left(\alpha \mathrm{Wr} S_{n}\right) \sigma \tau=\gamma \tau$. Next, consider the direct factorization $A \times A^{n-1}$ of the base group $A^{n}$, with the first direct factor $A$ being the coordinate subgroup indexed by the 'point' whose stabilizer was chosen as $S_{n-1}$ and the second direct factor $A^{n-1}$ being the product of all the other coordinate subgroups. Then $S_{n-1}$ centralizes the first direct factor and normalizes the second, so $S_{n-1} A^{n}$ is the direct product $A \times S_{n-1} A^{n-1}$; let $\tau_{0}$ stand for the corresponding idempotent endomorphism of $S_{n-1} A^{n}$ with image $A$ and kernel $S_{n-1} A^{n-1}$. Note that $H \gamma$ is contained in $S_{n-1} A^{n}$, so one may define $\alpha$ as $\alpha: h \mapsto h \gamma \tau_{0}$. Define $\varphi$ using coset representatives which may be arbitrary except for the trivial coset $H$ being represented by 1 , take a matching $\sigma$, and set $\gamma^{\prime}=\varphi\left(\alpha \mathrm{Wr} S_{n}\right) \sigma$. Appeal to the converse part of the 
Uniqueness Theorem of [8] with $a=1$ (noting that there the present $\tau$ and $\tau_{0}$ were called $\pi$ and $\pi_{0}$, respectively), and conclude that $\gamma^{\prime}$ is $\gamma$ followed by an inner automorhism, $\beta$ say (this one being conjugation by a suitable element of the base group). Then $\gamma=\varphi\left(\alpha \mathrm{Wr} S_{n}\right) \sigma \beta^{-1}$, and the proof is complete.

A proof of the part of Clifford's Theorem stated in the Introduction may now be sketched as follows. Suppose that $\rho\rfloor_{M}$ is not a direct sum of pairwise equivalent irreducible representations. Show that in this case $\rho$ is equivalent to a representation whose image lies in a block monomial subgroup $G L(d / n, \mathbb{F})$ Wr $S_{n}$ in such a way that the top projection maps it onto a transitive subgroup of $S_{n}$. The lemma then directly implies that $\rho$ is equivalent to a representation induced from a subgroup of index $n$. Thus one may argue that this lemma is just an abstract form of one step from 'the' proof of Clifford's Theorem. It is this sketch which will be followed in proving the main theorem of this paper as well.

REMARK. When $n$ is finite, any subgroup of index $n$ whose image under $\gamma$ normalizes a coordinate subgroup is the complete inverse image of a point stabilizer and thus can be chosen for the role of $H$.

\section{Outer tensor products}

In further preparation for the main theorem, some facts concerning outer tensor products also need to be reviewed. It will be convenient to do this in the language of linear groups instead of representations. The relevant fragment of the familiar result (see $[4,10 \mathrm{E}]$ ) may be elaborated as follows.

Outer Tensor Product Theorem. Let $K_{1}, \ldots, K_{n}$ be (not necessarily finite) subgroups of $G L(V)$ where $V$ is a finite dimensional vector space over an arbitrary field $\mathbb{F}$; suppose that $K_{i}$ and $K_{j}$ commute elementwise whenever $i \neq j$, and that the subgroup $K_{1} \cdots K_{n}$ is irreducible. For each $i$, choose an irreducible $K_{i}$-subspace $V_{i}$, and denote by $k_{i} \downarrow V_{i}$ the restriction to $V_{i}$ of an element $k_{i}$ of $K_{i}$. Suppose in addition that each $K_{i} \downarrow V_{i}$ is absolutely irreducible. Then there is a vector space isomorphism $\otimes V_{i} \cong V$ which conjugates each $\bigotimes\left(k_{i} \downarrow V_{i}\right)$ to the corresponding $\prod k_{i}$, and the subgroup $K_{1} \cdots K_{n}$ is absolutely irreducible.

NoRMALIZER ADDENDUM (EXTERNAL FORM). Let $N$ denote the intersection of the normalizers $\mathbb{N}_{G L(V)}\left(K_{i}\right)$ and $N_{i}$ the normalizer of the restriction $K_{i} \downarrow V_{i}$ in $G L\left(V_{i}\right)$ : then this $\otimes V_{i} \cong V$ conjugates $\otimes N_{i}$ to $N$. 
Since two isomorphisms $\otimes V_{i} \cong V$ which conjugate each $\otimes\left(k_{i} \downarrow V_{i}\right)$ to $\Pi k_{i}$ can differ only by a scalar factor, the abuse of language in referring to 'this' $\otimes V_{i} \cong V$ carries no danger. For an equivalent form of this addendum, let $Z$ stand for the centre of $G L(V)$ and $A_{i}$ for the linear span of $K_{i}$ in End $_{\mathrm{F}} V$ : note that, as (abstract) $\mathbb{F}$-algebra, $A_{i}$ is isomorphic to End $_{\mathrm{F}} V_{i}$. Further, let $L_{i}$ denote the normalizer of $K_{i}$ in the group of units, $G_{i}$ say, of $A_{i}$; of course, $Z \leq L_{i} \leq N$.

Normalizer AdDENDUM (INTERNAL FORM). The quotient $N / Z$ is the direct product of the $L_{i} / Z$.

Corollary 1. The quotient $N / Z$ is contained in the direct product of the $G_{i} / Z$.

Corollary 2. The quotient $N /\left(K_{1} \cdots K_{n} Z\right)$ is isomorphic to the direct product of the $L_{i} / K_{i} Z$.

Proof of the external form of the Normalizer Addendum. It will be convenient to write 1 , ambiguously but simply, for the identity transformation of every vector space. The proof will be by induction on $n$. The initial step is the critical one: the case $n=2$.

The key fact is that the normalizer in $G L\left(V_{1} \otimes V_{2}\right)$ of the algebra $1 \otimes \operatorname{End}_{\mathrm{F}} V_{2}$ is precisely $G L\left(V_{1}\right) \otimes G L\left(V_{2}\right)$. Indeed, $G L\left(V_{1}\right) \otimes G L\left(V_{2}\right)$ is obviously contained in that normalizer. As the simple modules for a full matrix algebra over $\mathbb{F}$ form a single isomorphism class, such an algebra has no outer automorphism: thus to each element $g$ of the normalizer there is a $y$ in $G L\left(V_{2}\right)$ such that $g\left(1 \otimes y^{-1}\right)$ centralizes $1 \otimes \operatorname{End}_{\mathrm{F}} V_{2}$. On the other hand, $V_{1} \otimes V_{2}$ as module for $1 \otimes \operatorname{End}_{\mathrm{F}} V_{2}$ is the direct sum of $\operatorname{dim} V_{1}$ pairwise isomorphic simple modules, so the endomorphism ring of this module has the same dimension as $\left(\right.$ End $\left._{F} V_{1}\right) \otimes 1:$ thus the centralizer of $1 \otimes \operatorname{End}_{F} V_{2}$ in $\operatorname{End}_{\mathbf{F}}\left(V_{1} \otimes V_{2}\right)$ is precisely $\left(\right.$ End $\left._{\mathbf{F}} V_{1}\right) \otimes 1$. It follows that $g\left(1 \otimes y^{-1}\right)=x \otimes 1$ for some $x$ in $G L\left(V_{1}\right)$, and then $g=x \otimes y$.

By a similar argument, the centralizer of $\left(K_{1} \downarrow V_{1}\right) \otimes 1$ in $\operatorname{End}_{\mathbf{F}}\left(V_{1} \otimes V_{2}\right)$ is just $1 \otimes$ End $_{\mathbf{F}} V_{2}$ : therefore $\mathbb{N}_{G L\left(V_{1} \otimes V_{2}\right)}\left(\left(K_{1} \downarrow V_{1}\right) \otimes 1\right)$ must normalize $1 \otimes \operatorname{End}_{\mathrm{F}} V_{2}$ as well, and so by the result of the previous paragraph it must lie in $G L\left(V_{1}\right) \otimes G L\left(V_{2}\right)$. Of course it contains the centralizer $1 \otimes G L\left(V_{2}\right)$ of $\left(K_{1} \downarrow V_{1}\right) \otimes 1$ in $G L\left(V_{1} \otimes V_{2}\right)$, so by Dedekind's Law

$$
\mathbb{N}_{G L\left(V_{1} \otimes V_{2}\right)}\left(\left(K_{1} \downarrow V_{1}\right) \otimes 1\right)=\left(N_{1} \otimes 1\right)\left(1 \otimes G L\left(V_{2}\right)\right)=N_{1} \otimes G L\left(V_{2}\right) .
$$

By symmetry we also have

$$
\mathbb{N}_{G L\left(V_{1} \otimes V_{2}\right)}\left(1 \otimes\left(K_{2} \downarrow V_{2}\right)\right)=G L\left(V_{1}\right) \otimes N_{2},
$$


so we can conclude that the intersection of the normalizers of $\left(K_{1} \downarrow V_{1}\right) \otimes 1$ and $1 \otimes\left(K_{2} \downarrow V_{2}\right)$ is $\left(N_{1} \otimes G L\left(V_{2}\right)\right) \cap\left(G L\left(V_{1}\right) \otimes N_{2}\right)$. As each $N_{i}$ contains all scalar transformations on the corresponding $V_{i}$, it follows that this intersection is $N_{1} \otimes N_{2}$, and the proof for $n=2$ is complete.

For the easy inductive step, let $n>2$. An element of $G L\left(\otimes V_{i}\right)$ which normalizes the copies of the $K_{i}$ (that is, the groups obtained as the Kronecker product of one $K_{i} \downarrow V_{i}$ with the identity tranformations on the $V_{j}$ with $j \neq i)$, will also normalize the product $\left(K_{1} \downarrow V_{2}\right) \otimes\left(K_{2} \downarrow V_{2}\right) \otimes 1 \otimes \cdots \otimes 1$ of the first two of these subgroups, and therefore by the inductive hypothesis it must be of the form $x \otimes y$ with $x \in G L\left(V_{1} \otimes V_{2}\right)$ and $y \in N_{3} \otimes \cdots \otimes N_{n}$. Since $1 \otimes y$ normalizes all relevant subgroups, so does $x \otimes 1$, and therefore $x$. must normalize both $\left(K_{1} \downarrow V_{1}\right) \otimes 1$ and $1 \otimes\left(K_{2} \downarrow V_{2}\right)$ : by the case $n=2$, therefore $x$ must lie in $N_{1} \otimes N_{2}$.

Proof of the internal form of the Normalizer Addendum. The case $i=1$ is typical: $\otimes V_{i} \cong V$ conjugates $\left(\right.$ End $\left._{F} V_{1}\right) \otimes 1 \otimes \cdots \otimes 1$ to $A_{1}$ and $N_{1} \otimes 1 \otimes \cdots \otimes 1$ to $L_{1}$.

\section{The other theorem of Clifford}

The 'other theorem of Clifford' referred to in the introduction says that if $\rho$ is a representation of a group $G$ and its restriction $\rho \downarrow_{M}$ to a normal subgroup $M$ is a direct sum of copies of an absolutely irreducible representation $\sigma$ of $M$, then there exist projective representations $\pi$ and $\tau$ of $G$ with $\tau \downarrow_{M}=\bar{\rho}$ and $\operatorname{ker} \pi \geq M$ such that $\bar{\sigma}=\tau \otimes \pi$. The input and output of this theorem are not matched in a way that would facilitate repeated application. Using the Normalizer Addendum, one can strengthen the conclusion and weaken the hypothesis so as to remedy this, as follows.

Some new terminology will be necessary, which will also be useful in the second version of the main result in the next section. Let us say that a projective representation $\tau: G \rightarrow P G L(V)$ and a (genuine) representation $\sigma: M \rightarrow G L(V)$ of a normal subgroup $M$ of $G$ are linked if $\left(m^{g}\right) \sigma=(m \sigma)^{x}$ whenever $m \in M, g \in G$, and $x$ is a preimage of $g \tau$ in $G L(V)$. For example, this is the case when $\tau=\bar{\rho}$ and $\sigma=\rho \downarrow_{M}$ for some representation $\rho$ of $G$. If $\tau$ and $\sigma$ are linked then of course $\sigma$ is $G$-invariant (equivalent to all its $G$-conjugates).

THE OTHER THEOREM OF CLIFFORD (EXTENDED FORM). To each G-invariant absolutely irreducible representation $\sigma$ of a normal subgoup $M$ of $G$, there is a unique $\tau$ such that $\sigma$ and $\tau$ are linked; then $\tau \downarrow_{M}=\bar{\sigma}$ (so $\tau$ is absolutely irreducible) and $\operatorname{ker} \tau=\mathbb{C}_{G}(M / \operatorname{ker} \sigma)$. If $\sigma^{\prime}$ is equivalent to such 
$a \sigma$, then the unique $\tau^{\prime}$ linked to $\sigma^{\prime}$ is equivalent to $\tau$. If $\pi$ is a projective representation of $G$ which is linked to a direct sum of copies of such $a \sigma$, then the $\tau$ linked to $\sigma$ is a tensor factor of $\pi$.

(Of course the complementary tensor factor is then also unique, for $\tau \otimes$ $\pi^{\prime}=\tau \otimes \pi^{\prime \prime}$ is impossible without $\pi^{\prime}=\pi^{\prime \prime}$.)

The proof of the first two sentences is obvious. For the third, apply the Normalizer Addendum with $n=2$ : let $\rho$ be the relevant direct sum of copies of $\sigma$; let $K_{1}=M \rho$, so $m \mapsto\left(m \rho \downarrow V_{1}\right)$ can be identified with $\sigma$; and put $K_{2}=\mathbb{C}_{G L(V)}\left(K_{1}\right)$. Show that the irreducible constituents of $K_{2}$ are absolutely irreducible and that $K_{1} K_{2}$ is irreducible. The conclusion is that $G \pi \leq\left(G_{1} / Z\right) \times\left(G_{2} / Z\right)$. Following $\pi$ by the projections of this direct product onto its direct factors, one obtains two projective representations whose tensor product is $\pi$. The first tensor factor is then readily seen to be linked to $\sigma$, so it must equal $\tau$.

Remarks. A few caveats may be in order, even for the case of finite $G$ and faithful, absolutely irreducible, $G$-invariant $\sigma$. First, $\tau^{\prime} \downarrow_{M}=\bar{\sigma}$ does not imply that $\operatorname{ker} \tau^{\prime}=\mathbb{C}_{G}(M)$. Second, even if $\operatorname{ker} \tau^{\prime}=\mathbb{C}_{G}(M)$ is also assumed, one cannot conclude in general that $\tau^{\prime}$ is equivalent to $\tau$. (Examples in Section 8 will show the need for these comments.) Third, it must be emphasized that even if $\tau^{\prime}$ is equivalent to $\tau$, it is not linked to $\sigma$ unless it actually equals $\tau$ (otherwise it is linked to a representation equivalent to but distinct from $\sigma$ ). Similarly, the uniqueness of the complementary tensor factor (in the parenthetical remark after the theorem) is not just 'up to equivalence'. Thus in this context extreme caution is needed with otherwise harmless abuses of language, like saying 'is induced (or tensor induced)' while meaning 'is equivalent to an induced (or tensor induced) representation (or projective representation)'.

As there is no nontrivial 1-dimensional projective representation (in the sense of this paper), no projective representation can be a tensor factor of a strictly different projective representation of the same dimension. Thus the first caveat already shows that, in [1, Corollary 5.19] for example, the assumption that a restriction of a projective representation 'is' genuine (written there as " $\left.Y\right|_{R}$ is nonprojective") must be interpreted as requiring that the projective representation be linked to the relevant genuine representation.

\section{The main result}

Reverting to those 'harmless abuses of language', recall that a (genuine) representation is called primitive if it is irreducible and not induced from 
any proper subgroup. Similarly, a projective representation will be called tensor primitive if it is irreducible, not a tensor product (of projective representations of dimension greater than 1), and not tensor induced (from any proper subgroup).

TENSOR INDUCTION THEOREM. Let $G$ be a (not necessarily finite) group with a faithful primitive representation $\rho$. Suppose that $\bar{\rho}$ is not a tensor product (of projective representations of dimension greater than 1 ). Let $K$ be a noncentral subgroup which centralizes all its conjugates in $G$ except perhaps itself, set $\mathbb{N}_{G}(K)=H$, and suppose that some irreducible constituent, $\sigma$ say, of $\rho \downarrow_{K}$ is absolutely irreducible. Then ( $\rho$ is absolutely irreducible and) $\bar{\rho}$ is tensor induced from a projective representation of $H$, namely from a tensor factor $\pi$ of $\bar{\rho}\rfloor_{H}$ such that $\left.\pi\right\rfloor_{K}=\bar{\sigma}$ and $\operatorname{ker} \pi=\mathbb{C}_{G}(K)$.

Proof. Denote the index $|G: H|$ by $n$; set $K_{1}=K$, let $K_{1}, \ldots, K_{n}$ be the conjugates of $K$ in $G$, and $M$ the subgroup generated by the $K_{i}$. This $M$ is a noncentral normal subroup of $G$. The primitivity of $\rho$ ensures that the irreducible constituents of $\rho \downarrow_{M}$ are pairwise equivalent, by the part of Clifford's Theorem discussed above. Each $K_{i}$ is supplemented by its centralizer in $M$, so another application of (another part of) Clifford's Theorem now yields that the irreducible constituents of $\rho \downarrow_{K_{i}}$ are pairwise equivalent. By assumption then, all these constituents are absolutely irreducible. Therefore the Outer Tensor Product Theorem shows that the irreducible constituents of $\rho \downarrow_{M}$ are also absolutely irreducible. Since $\bar{\rho}$ is not a tensor product, it follows that $\rho \downarrow_{M}$ itself is absolutely irreducible (use the original version of 'the other theorem of Clifford'). Of course $H$ contains $M$, so $\rho\rfloor_{H}$ is also absolutely irreducible. Since $K$ is not normal in $G$ and $\rho$ is faithful, $K \rho$ cannot consist of scalars and so $K$ cannot be central in $H$. The scene is now set for applying the extended form of 'the other theorem of Clifford' with $H, \bar{\rho} \downarrow_{H}, K$, and $\sigma$ in place of $G, \pi, M$, and $\sigma$, leading to the conclusion that $\bar{\rho} \downarrow_{H}$ is the tensor product of two projective representations of $H$, the first of which, $\tau$ say, has kernel $\mathbb{C}_{G}(K)$ and agrees with $\bar{\sigma}$ on $K$. It remains to show that $\bar{\rho}$ is equivalent to $\tau \uparrow^{\otimes G}$.

Set $C=\mathbb{C}_{G L(d, \mathbf{F})}(K \rho)$ and let $G_{i}$ denote the group of units in the linear span of $K_{i} \rho$. Write $Z$ for the centre of $G L(d, \mathbb{F})$, and $N$ for the intersection of the normalizers of $K \rho$ and of $C$ in $G L(d, \mathbb{F})$. Corollary 1 (in Section 4) shows that $H \bar{\rho} \leq N / Z \leq\left(G_{1} / Z\right) \times(C / Z)$; by definition, $\tau$ is $\bar{\rho} \downarrow_{H}$ followed by the projection of this direct product onto its first direct factor. Of course now the $G_{i} / Z$ are the coordinate subgroups of the base group of a copy of $P G L(\sqrt[n]{d}, \mathbb{F}) \mathrm{Wr} S_{n}$ in $P G L(d, \mathbb{F})$, a copy whose inclusion, $\widetilde{\kappa}$ say, is obtained from Kronecker multiplication, just as in the 
definition of tensor induction of projective representations. That copy, $W$ say, may be recognized as the normalizer in $P G L(d, \mathbb{F})$ of the set consisting of these coordinate subgroups, and hence it contains $G \bar{\rho}$. Indeed, since the $K_{i}$ are all conjugate in $G$, the 'top projection' of this wreath product maps $G \bar{\rho}$ onto a transitive subgroup of $S_{n}$. The lemma of $\S 3$ therefore ensures that (up to equivalence) $\bar{\rho}$ is tensor induced from a subgroup of index $n$. As $H \bar{\rho}$ normalizes one of the coordinate subgroups and $H$ has index $n$, the remark at the end of Section 3 shows that the present $H$ can be chosen as that subgroup. The definition of $\alpha$ in the proof of that lemma now amounts to naming a projective representation of $H$ which tensor induces to (a projective representation equivalent to) $\bar{\rho}$, as follows. Let $S_{n-1}$ be chosen so that $S_{n-1} \widetilde{\kappa}$ is the normalizer of $G_{1} / Z$ in $S_{n} \widetilde{\kappa}$. Then $\mathbb{N}_{W}\left(G_{1}\right)=\left(G_{1} / Z\right) \times\left(\left(S_{n-1} \widetilde{\kappa}\right)\left(G_{2} / Z\right) \cdots\left(G_{n} / Z\right)\right)$, and $\alpha$ is to be $\bar{\rho} \downarrow_{H}$ followed by the projection, $\pi_{0}$ say, of this direct product onto its first direct factor. The second direct factor being contained in $C / Z$, this $\pi_{0}$ is just a restriction of the projection of $\left(G_{1} / Z\right) \times(C / Z)$ onto its first direct factor. This shows that the definition of $\alpha$ matches that of $\tau$, and thereby completes the proof.

REMARK: If $\rho: G \rightarrow G L(d, \mathbb{F})$ is a representation such that $\bar{\rho}=\tau \uparrow^{\otimes G}$ for some projective representation $\tau$ of a subgroup $H$ of index $n$, then (in the notation of Section 2) one has that

$$
G \rho \leq\left(G L(\sqrt[n]{d}, \mathbb{F}) \mathrm{Wr} S_{n}\right) \kappa^{+},
$$

so the pullback of $\rho$ and $\kappa^{+}$gives a surjective homomorphism $\rho^{*}: G^{*} \rightarrow G$ such that $\rho^{*} \rho$ factors also through $\kappa^{+}$. The top projection of this wreath product must then map the relevant image of $G^{*}$ to a transitive subgroup of $S_{n}$, and it readily follows that $\rho^{*} \rho=\sigma \uparrow^{\otimes G^{*}}$ for some representation $\sigma: H^{*} \rightarrow G L(\sqrt[n]{d}, \mathbb{F})$ of the complete inverse image $H^{*}$ of $H$ under $\rho^{*}$. (Moreover, here $\left(x \rho^{*}\right) \tau=x \bar{\sigma}$ for all $x$ in $H^{*}$. Note however that $\operatorname{ker} \rho^{*}$ is usually not central in $H^{*}$, let alone in $G^{*}$.) In this very weak sense only, $\rho$ may be viewed as a tensor induced genuine representation, not of $G$ but of the larger group $G^{*}$. (The first example in Section 8 will show that $\rho$ itself need not be tensor induced when the hypotheses of the Tensor Induction Theorem are satisfied.) The same argument may be applied even when a projective representation $\pi=\tau \uparrow^{\otimes G}$ tensor induced from a subgroup $H$ is not (or indeed cannot be) written in the form $\bar{\rho}$ (for any genuine representation $\rho$ of $G$ ): the pullback of $\tau \uparrow^{\otimes G}$ and the relevant $\overline{\kappa^{+}}$yields a surjective homomorphism $\pi^{*}: G^{*} \rightarrow G$ such that $\pi^{*} \pi=\overline{\sigma^{\otimes G^{*}}}$ for a suitable genuine representation $\sigma$ of the complete inverse image $H^{*}$, with $\left(x \pi^{*}\right) \tau=x \bar{\sigma}$ for all $x$ in $H^{*}$. 
COROllary. Let $G$ be a (not necessarily finite) group and $\rho$ a faithful primitive representation of $G$ such that $\bar{\rho}$ is tensor primitive. If $M$ is a noncentral normal subgroup of $G$ such that some irreducible constituent of $\rho \downarrow_{M}$ is absolutely irreducible, then $\rho \downarrow_{M}$ is in fact irreducible and $\mathbb{C}_{G}(M)=Z(G)$. If $K$ is a nonnormal subgroup of $G$ such that some irreducible constituent of $\rho \downarrow_{K}$ is absolutely irreducible, then some conjugate of $K$ in $G$, other than $K$ itself, must fail to centralize $K$.

(Of course the first part of this omnibus corollary comes direct from Clifford's theorems and has nothing to do with tensor induction.)

The chosen form of the Tensor Induction Theorem is open to objection: Clifford reduction leads to projective representations which do not come as $\bar{\rho}$ and which may not be capable of being written as such for any genuine representation $\rho$ of the group in question; in any case, it is incongruous that the hypotheses should involve genuine representations when the conclusions refer only to projective representations. The way to overcome these points is to make full use of the extended form of 'the other theorem of Clifford' from Section 5 (and avoid those 'harmless abuses').

TENSOR INDUCTION THEOREM (EXTENDED FORM). Let $\pi$ be a projective representation of a (not necessarily finite) group $G$, and $K$ a noncentral subgroup of $G$ which centralizes all its conjugates except perhaps itself. Write $H$ for the normalizer of $K$ and $M$ for the normal closure of $K$. Suppose that $\pi$ is linked to some faithful irreducible representation $\rho$ of $M$, and that an irreducible constituent $\sigma$ of $\rho \downarrow_{K}$ is absolutely irreducible. Then ( $\rho$ is absolutely irreducible and) $\pi$ is equivalent to a projective representation tensor induced from the unique projective representation $\tau$ of $H$ which is linked to $\sigma$ (and is therefore such that $\operatorname{ker} \tau=\mathbb{C}_{G}(K)$ and $\tau \downarrow_{K}=\bar{\sigma}$ ).

Modulo 'the other theorem of Clifford', this may be viewed as a (partial) generalization of [1, Corollary 5.19]; it certainly would not have been thought of without that result of Berger's.

\section{An application}

Suppose now that $G$ is a finite nonabelian group with a faithful primitive representation $\rho$, over a field $\mathbb{F}$ which is a splitting field for all quasinilpotent subnormal subgroups of $G$, and that $\bar{\rho}$ is tensor primitive. This immediately implies that the centre of $G$ is cyclic: call that $Z$, write $F$ for the generalized Fitting subgroup $F^{*}(G)$, and note that $\mathbb{C}_{G}(F) \leq F$ (see $[7, \mathrm{X} .13]$ ). 
There are two cases. First, let $G$ have a component $K$ (that is, a quasisimple subnormal subgroup). Then all conjugates of $K$ are also components of $G$, so those (if any) which are different from $K$ centralize $K$. By the corollary of the Tensor Induction Theorem, $K$ must be normal in $G$ and centralized only by $Z$ : thus $K \cap Z=Z(K)$ and $K Z=F$, whence $F / Z$ is isomorphic to the simple group $K / Z(K)$.

The rest of the argument concerns the alternative, namely the case in which $G$ has no component, so $F$ is just the Fitting subgroup. Since $G$ is nonabelian, $F$ cannot be central in $G$, so by (the Clifford part of) that corollary $F$ is also nonabelian. Let $M$ be minimal among the noncentral normal subgroups of $G$ contained in the second centre of $F$ : for the same reason, $\mathbb{C}_{G}(M)=Z$; in particular, $M \cap Z=Z(M)$. Thus $\mathbb{C}_{G}(M / Z(M))$ is nilpotent (for instance, by the 'three subgroups lemma'), and it follows that $\mathbb{C}_{G}(M / Z(M))=F$. For each element $f$ of $F$, define a map $\tilde{f}: M / Z(M) \rightarrow$ $Z$ by $\tilde{f}: x Z(M) \mapsto[x, f]$. It is straightforward to check, first, that each $\widetilde{f}$ so defined is a homomorphism, and second, that $f \mapsto \widetilde{f}$ defines a homomorphism of $F$ into $\operatorname{Hom}(M / Z(M), Z)$ with kernel $\mathbb{C}_{F}(M)$, that is, with kernel $Z$. Now $|\widetilde{M}|=|M / Z(M)| \geq|\operatorname{Hom}(M / Z(M), Z)| \geq|\widetilde{F}|$ (the reason for the first inequality being that $Z$ is cyclic), so in fact $M Z=F$ and $F / Z$ is $G$-isomorphic to $M / Z(M)$. We have already seen, in effect, that $M / Z(M)$ is a faithful irreducible $G / F$-module: so the same holds for $F / Z$.

Let $p$ be the (prime) exponent of $M / Z(M)$ : of course now $M$ has $p$ power order; the derived group $M^{\prime}$ has exponent $p$ and lies in the cyclic centre, so $M^{\prime}$ is the unique subgroup of order $p$ in $Z(M)$. If $M$ has no other subgroup of order $p$, then $M$ must be a quaternion group of order 8. If $M$ does have noncentral subgroups of order $p$ as well, the minimal choice of $M$ guarantees that the subgroup generated by the elements of order $p$ in $M$ must be $M$ itself. It follows that $M$ is either an extraspecial group (of exponent $p$ when $p$ is odd) or the central product of an extraspecial 2-group with a cyclic group of order 4. (An example in the next section will show that the latter possibility cannot be ruled out in general. It certainly cannot occur when $G$ is soluble, for in that case a minimal normal subgroup, $Q / F$ say, of $G / F$ must have order prime to $p$ and therefore $M / M^{\prime}$ is the direct product of $[M, Q] / M^{\prime}$ and $Z(M) / M^{\prime}$, so the minimal choice of $M$ implies that $[M, Q]=M$ and then $Z(M)=M^{\prime}$.) As usual, commutators yield a $G$-invariant nondegenerate symplectic form on $M / Z(M)$; when $p=$ $2=|Z(M)|$, one must consider also the $G$-invariant nondegenerate quadratic form obtained from squaring (see [6, III.13]).

Consider the possibility that $M / Z(M)$ as $G / F$-module is induced from a proper subgroup, $H / F$ say. This would mean that $H$ is the normalizer 
in $G$ of a subgroup $K$ with $M>K>Z(M)$ and $M / Z(M)$ is the direct product of the conjugates of $K / Z(M)$. In the terminology of Berger [1], form induction would mean in addition that any two distinct conjugates of $\mathrm{K}$ centralize one another. The corollary of the Tensor Induction Theorem prohibits this, so the conclusion is that $M / Z(M)$ is form primitive. In view of the $G$-isomorphism $F / Z \cong M / Z(M)$, this completes the proof of the claims made in the Introduction.

\section{Examples}

1. Consider the subgroup $G$ generated in $G L(4,3)$ by the Kronecker product of two copies of $S L(2,3)$ and a permutation matrix $g$ of order 2 which interchanges the two Kronecker factors, and let $\rho$ be the inclusion of $G$ in $G L(4,3)$ viewed as a representation over the algebraic closure $\mathbb{F}$ of the field of 3 elements. This group $G$ has no subgroup of index 4 . The unique subgroup of index 2 has no representation of dimension 2 which would not be trivial on the centre. Consequently, no faithful 4-dimensional genuine representation of $G$ can be induced or tensor induced from any proper subgroup. The image of $\bar{\rho}$ has a minimal normal subgroup of order 16; if $\bar{\rho}$ is written as a tensor product, its image is embedded in a direct product, and this minimal normal subgroup must survive projection into at least one of the direct factors. If that tensor factorization were nontrivial, the direct factors would be copies of $\operatorname{PGL}(2, \mathbb{F})$, but this group has no elementary abelian subgroup of order 16 . For $K$, take one of the Kronecker factors $S L(2,3)$. Then all hypotheses of the Tensor Induction Theorem are satisfied, yet $\rho$ is not tensor induced.

2. The same group $G$ also has a 4-dimensional faithful absolutely irreducible representation $\rho$ over the rational field $\mathbb{Q}$. In that case, $\rho \downarrow_{K}$ is irreducible but not absolutely irreducible; all other hypotheses are satisfied; yet $\bar{\rho}$ is not tensor induced. This shows that the assumption concerning an irreducible constituent of $\rho \downarrow_{K}$ being absolutely irreducible cannot be replaced by the requirement that $\rho$ itself be absolutely irreducible.

3. In the notation of Bolt, Room, Wall [3], let $G$ be the commutator subgroup $C T^{\prime}$ of the normalizer $C T$ in $G L(4, \mathbb{C})$ of the 4-dimensional 'complex Clifford group' $\mathscr{E} \mathscr{G}$. Then $G$ is a perfect group; its Fitting subgroup $F$ is $\mathscr{C} \mathscr{G}$, a central product of an extraspecial group of order 32 and a cyclic group $Z$ of order 4 ; this $Z$ is the centre of $G$, and $G / Z$ is a semidirect product of the self-centralizing minimal normal subgroup $F / Z$ with an alternating group $A_{6}$. As any perfect group, $G$ has no subgroup of 
index 2 or 4: so its inclusion $\rho: G \rightarrow G L(4, \mathbb{C})$ has nowhere to be induced from, and the corresponding $\bar{\rho}$ cannot be tensor induced either. The argument concerning the first example can now be repeated to show that $\bar{\rho}$ is not a tensor product. Nevertheless, $G$ has no extraspecial normal subgroup: it cannot possibly have one, for $A_{6}$ is too big to fit into the automorphism group of any extraspecial group of order 8 or 32 .

It is one of the less well known achievements of Professor Wall that the automorphism groups of the extraspecial groups were in effect determined already in the papers [2], [3] (except for the case of odd prime square exponent). The context dictated that the discussion be restricted to automorphisms which act trivially on the centre, on the other hand, the delicate issue of just when does the group of such automorphisms split over the group of inner automorphisms, was completely resolved.

4. The last example may also be used to illustrate the need for the second caveat in the remark at the end of Section 5 as follows. Since

$$
H^{1}(G \bar{\rho} / F \bar{\rho}, F \bar{\rho}) \neq 0
$$

(see Holt and Plesken [5]), the centralizer $\mathbb{C}_{\text {Aut }(G \bar{\rho})}(F \bar{\rho})$ contains some outer automorphisms (see Robinson [10, Section 4]): let $\alpha$ be one. Then $\tau^{\prime}: g \mapsto$ $(g \bar{\rho}) \alpha$ defines a projective representation $\tau^{\prime}$ of $G$ such that $\left.\tau^{\prime} \downarrow_{F}=\bar{\rho}\right\rfloor_{F}$ and $\operatorname{ker} \tau^{\prime}=\operatorname{ker} \bar{\rho}=Z$. The equivalence of $\tau^{\prime}$ and $\bar{\rho}$ would mean that $\alpha$ is conjugation by an element, $x$ say, of $\operatorname{PGL}(4, \mathbb{C})$. That $x$ would normalize $G \bar{\rho}$ and centralize $F \bar{\rho}$, so a preimage $y$ of $x$ in $G L(4, \mathbb{C})$ would normalize the complete inverse image $C G$ of $F \bar{\rho}$ : thus $y \in C T$ and so $x \in P C T$ would follow. Since $F \bar{\rho}=P C G$ and $P C G$ is its own centralizer in $P C T$, this would mean that $x \in F \bar{\rho}$ : but that would make $\alpha$ inner. This proves that $\tau^{\prime}$ and $\bar{\rho}$ are not equivalent.

5. The first caveat in that remark is much easier to justify. For this, let $G$ be the holomorph of the quaternion group $Q_{8}$ of order 8 . The group $R$ of the right tranlations and the group $L$ of the left translations are normal subgroups of $G$, with Aut $Q_{8}$ a common complement to them. There is just one other normal subgroup of order 8 in $G$, namely the elementary abelian $A$ defined by $A=(R \cap L)\left(\operatorname{Inn} Q_{8}\right)$; and all three factor groups of order 24 are isomorphic to $P G L(2,3)$. It follows that there exist homomorphisms $\tau$ and $\tau^{\prime}$ of $G$ onto $\operatorname{PGL}(2,3)$ with kernels $A$ and $L$, respectively; and it is not hard to arrange that $\tau$ and $\tau^{\prime}$ agree on $R$. 


\section{References}

[1] T. R. Berger, 'Hall-Higman type theorems V,' Pacific J. Math. 73 (1977), 1-62.

[2] Beverly Bolt, T. G. Room, and G. E. Wall, 'On the Clifford collineation, transform and similarity groups. I,' J. Austral. Math. Soc. 2 (1961-1962), 60-79.

[3] Beverly Bolt, T. G. Room, and G. E. Wall, 'On the Clifford collineation, transform and similarity groups. II,' J. Austral. Math. Soc. 2 (1961-1962), 80-96.

[4] Charles W. Curtis and Irving Reiner, Methods of representation theory I, Wiley, New York, Chichester, Brisbane, Toronto, 1981.

[5] Derek F. Holt and W. Plesken, Perfect groups, Clarendon Press, Oxford, 1989.

[6] B. Huppert, Endliche Gruppen I, Springer-Verlag, Berlin, Heidelberg, New York, 1967.

[7] B. Huppert and N. Blackburn, Finite groups III, Springer-Verlag, Berlin, Heidelberg, New York, 1982.

[8] L. G. Kovács, 'Two results on wreath products,' Arch. Math. 45 (1985), 111-115.

[9] L. G. Kovács, 'Some theorems on wreath products,' Publ. Math. Debrecen 35 (1988), 155-160.

[10] D. J. S. Robinson, 'Applications of cohomology to the theory of groups,' in Groups-St. Andrews 1981, ed. by C. M. Campbell and E. F. Robertson, London Math. Soc. Lecture Notes Ser. 71, Cambridge University Press, Cambridge, 1982, pp. 46-80.

\section{Mathematics IAS}

Australian National University

GPO Box 4

Canberra 2601

Australia 